\title{
Stability for Functional Differential Equations with Delay in Banach Spaces
}

\author{
Anna Kisiolek ${ }^{1}$, Ireneusz Kubiaczyk ${ }^{1}$, Aneta Sikorska-Nowak ${ }^{2}$ \\ ${ }^{1}$ The Great Poland University of Social and Economics in Środa Wlkp, Środa Wlkp, Poland \\ ${ }^{2}$ Faculty of Mathematics and Computer Science, Adam Mickiewicz University, Poznań, Poland \\ Email: akisiolek@wp.pl,kuba@amu.edu.pl, an etas@amu.edu.pl
}

How to cite this paper: Kisiolek, A., Kubiaczyk, I. and Sikorska-Nowak, A. (2019) Stability for Functional Differential Equations with Delay in Banach Spaces. $A d$ vances in Pure Mathematics, 9, 337-346. https://doi.org/10.4236/apm.2019.94016

Received: March 14, 2019

Accepted: April 25, 2019

Published: April 28, 2019

Copyright (c) 2019 by author(s) and Scientific Research Publishing Inc. This work is licensed under the Creative Commons Attribution International License (CC BY 4.0).

http://creativecommons.org/licenses/by/4.0/

\begin{abstract}
In this paper, using Sadovskii's fixed point theorem and properties of the measure of noncompactness, we obtain asymptotic stability results for solutions of nonlinear differential equation with variable delay. Results presented in this paper extend some previous results due to Burton [1], Becker and Burton [2], Ardjouni and Djoudi [3], and Jin and Luo [4].
\end{abstract}

\section{Keywords}

Fixed Point, Differential Equations, Banach Space, Stability, Measure of Noncompactness

\section{Introduction}

Since 1892, the Lyapunov's direct method has been used for the study of stability properties of ordinary, functional, differential and partial differential equations. Nevertheless, the applications of this method to problem of stability in differential equations with delay have encountered serious difficulties if the delay is unbounded or if the equation has unbounded terms (see [1] [5] [6]). Recently, investigators, such as Burton and Furumochi have noticed that some of these difficulties vanish or might be overcome by means of fixed point theory (see [1]-[17]). The fixed point theory does not only solve the problem on stability but has a significant advantage over Lyapunov's direct method. Sadovskii's fixed point theorem (see [18]) and techniques of the theory of the measure of noncompactness are used to prove the existence and stability of the solution of the problem investigated in this paper.

Let $E$ denote a Banach space. We consider the nonlinear differential equation with variable delay 


$$
x^{\prime}(t)=-a(t) x\left(t-r_{1}(t)\right)+b(t) x^{\prime}\left(t-r_{1}(t)\right)+c(t) G\left(x(t), x\left(t-r_{2}(t)\right)\right)
$$

with the initial condition

$$
x(t)=\psi(t), \text { for } t \in[m(0), 0],
$$

where $\psi \in C([m(0), 0], E), m_{j}(0)=\inf \left\{t-r_{j}(t), t \geq 0\right\}$, $m(0)=\min \left\{m_{j}(0), j=1,2\right\}$.

Here $C B\left(R^{+}, E\right)$ denotes the set of all continuous and bounded functions $f: R^{+} \rightarrow E$ with the supremum norm $\|\cdot\|$ defined

$\|x\|=\sup \{\|x(t)\|: m(0) \leq t<\infty\}$. Throughout this paper, we assume that $a, b, c \in C B^{1}\left(R^{+}, R\right), \quad r_{1} \in C^{2}\left(R^{+}, R\right), r_{2} \in C\left(R^{+}, R\right) \quad$ with $\quad t-r_{j}(t) \rightarrow \infty \quad$ as $t \rightarrow \infty, j=1,2$. We also assume that the function $G: E \times E \rightarrow E$ is uniformly continuous. Moreover, we assume that the function $G$ is bounded, so there exists a constant $L \geq 0$, such that $\|G(\cdot, \cdot)\| \leq L$ and $G(0,0)=0$.

Special cases of the equation (1) have been investigated by many authors. For example, Burton in [6] and Zhang in [17] have studied boundedness and stability of the linear equation:

$$
x^{\prime}(t)=-a(t) x\left(t-r_{1}(t)\right) .
$$

In [13], Burton and Furumochi using the fixed point theorem of Krasnosielski obtained boundedness and asymptotic stability for the equation:

$$
x^{\prime}(t)=-a(t) x\left(t-r_{1}\right)+b(t) x^{\gamma}\left(t-r_{2}(t)\right),
$$

where $r_{1}>0$ is constant; $\gamma \in(0,1)$ and $\gamma$ is a quotient with odd positive integer denominator and $a \in C\left(R^{+},(0, \infty)\right)$.

Next, Jin and Luo (see [4]), proved the boundedness and stability of solutions of the equation

$$
x^{\prime}(t)=-a(t) x\left(t-r_{1}(t)\right)+b(t) x^{1 / 3}\left(t-r_{2}(t)\right)
$$

and generalized the results claimed in [6] [12] [17]. Ardjouni and Djoudi in [3] considered the more general neutral nonlinear differential equation

$$
x^{\prime}(t)=-a(t) x\left(t-r_{1}(t)\right)+b(t) x^{\prime}\left(t-r_{1}(t)\right)+c(t) G\left(x^{\gamma}\left(t-r_{2}(t)\right)\right)
$$

and obtained the boundedness and stability results.

We mentioned here that the neutral delay differential equations appear in modelling of the networks containing lossless transmission lines (as in high-speed computers where the lossless transmission lines are used to interconnect switching circuits), in the study of vibrating masses attached to an elastic bar, as the Euler equation in some variational problems, theory of automatic control and in neuromechanical systems in which inertia plays an important role, we refer the reader to the papers by Boe and Chang [19], Brayton and Willoughby [20] and to the books by Driver [21], Hale [14] and Popov [22] and reference cited therein.

The fundamental tool in this paper is the Kuratowski measure of noncompactness (see [23]). 
For any bounded subset $A$ of $E$ we denote by $\alpha(A)$ the Kuratowski measure of noncompactness of $A$, i.e. the infimum of all $\epsilon>0$ such that there exists a finite covering of $A$ by sets of diameters smaller than $\epsilon$. The properties of the measure of noncompactness $\alpha$ are:

1) if $A \subset B$ then $\alpha(A) \leq \alpha(B)$;

2) $\alpha(A)=\alpha(\bar{A})$, where $\bar{A}$ denotes the closure of $A$;

3) $\alpha(A)=0$ if and only if $A$ is relatively compact;

4) $\alpha(A \cup B)=\max \{\alpha(A), \alpha(B)\}$

5) $\alpha(\lambda A)=|\lambda| \alpha(A),(\lambda \in R)$;

6) $\alpha(A+B) \leq \alpha(A)+\alpha(B)$

7) $\alpha(\operatorname{conv} A)=\alpha(A)$, where $\operatorname{conv}(A)$ denotes the convex hull of $A$,

8) $\alpha(A)<\delta(A)$, where $\delta(A)=\sup _{x, y \in A}\{\|x-y\|\}$.

Lemma 1. [24]. Let $K \subset C(I, E)$ be a family of strongly equicontinuous functions. Let $K(t)=\{k(t) \in E, k \in K\}$, for $t \in I$ and $K(I)=\bigcup_{t \in I} K(t)$. Then

$$
\alpha_{C}(K)=\sup _{t \in I} \alpha(K(t))=\alpha(K(I))
$$

where $\alpha_{C}(K)$ denotes the measure of noncompactness in $C(I, E)$ and the function $t \mapsto \alpha(K(t))$ is continuous.

Let us denote by $S_{\infty}$ the set of all nonnegative real sequences. For $\xi=\left(\xi_{n}\right) \in S_{\infty}, \quad \eta=\left(\eta_{n}\right) \in S_{\infty}$, we write $\xi<\eta$ if $\xi_{n} \leq \eta_{n}$ (i.e. $\xi_{n}<\eta_{n}$, for $n=1,2, \cdots)$ and $\xi \neq \eta$.

Let $X$ be a closed convex subset of $C(I, E)$ and let $\Phi$ be a function which assigns to each nonempty subset $Z$ of $X$ a sequence $\Phi(Z) \in S_{\infty}$ such that

$$
\begin{gathered}
\Phi(\{z\} \cup Z)=\Phi(Z), \text { for } z \in X, \\
\Phi(\overline{\operatorname{conv}} Z)=\Phi(Z),
\end{gathered}
$$

if $\Phi(Z)=\Theta$ (the zero sequence), then $\bar{Z}$ is compact.

In the proof of the main theorem, we will apply the following fixed point theorem

Theorem 1 [18] If $F: X \rightarrow X$ is continuous mapping satisfying $\Phi(F(Z))<\Phi(Z)$ for arbitrary nonempty subset $Z$ of $X$ with $\Phi(Z)>0$, then $F$ has a fixed point in $X$.

\section{Main Results}

A solution of the problem (1) is a continuous function $x:[m(0), \infty) \rightarrow E$ such that $x$ satisfies (1) on $[0, \infty)$ and $x=\psi$ on $[m(0), 0]$. Stability definitions may found in [1], for example.

In this paper, we extend stability theorem proved in [3] by giving a necessary and sufficient conditions for asymptotic stability of the zero solution of the Equation (1). By using some conditions expressed in terms of the measure of noncompactness which $G$ satisfies, we define a continuous, bounded operator $P_{\phi}$ over the Banach space $S_{\psi}$, whose fixed points are solutions of (1). The fixed 
point theorem of Sadovskii is used to prove the existence of a fixed point of the operator $P_{\phi}$. To construct our mapping, we begin transforming (1) to a more tractable, but equivalent equation, which we invert to obtain an equivalent integral equation for which we derive a fixed point mapping. We need the following lemma in our proof of the main theorem.

Lemma 2 Let $g:[m(0), \infty) \rightarrow R^{+}$be an arbitrary continuous function and suppose that

$$
r_{1}^{\prime}(t) \neq 1, \forall t \in R^{+}
$$

Then $x$ is a solution of $(1)$ if and only if

$$
\begin{aligned}
x(t)= & \left(x(0)-\frac{b(0)}{1-r_{1}^{\prime}(0)} x\left(-r_{1}(0)\right)-\int_{-r_{1}(0)}^{0} g(u) x(u) \mathrm{d} u\right) \mathrm{e}^{-\int_{0}^{t} g(u) \mathrm{d} u} \\
& +\frac{b(t)}{1-r_{1}^{\prime}(t)} x\left(t-r_{1}(t)\right)+\int_{t-r_{1}(t)}^{t} g(u) x(u) \mathrm{d} u \\
& -\int_{0}^{t} \mathrm{e}^{-\int_{s}^{t} g(u) \mathrm{d} u} g(s)\left(\int_{s-r_{1}(s)}^{s} g(u) x(u) \mathrm{d} u\right) \mathrm{d} s \\
& +\int_{0}^{t} \mathrm{e}^{-\int_{s}^{t} g(u) \mathrm{d} u}\left[g\left(s-r_{1}(s)\right)\left(1-r_{1}^{\prime}(s)\right)-a(s)-\mu(s)\right] x\left(s-r_{1}(s)\right) \mathrm{d} s \\
& +\int_{0}^{t} \mathrm{e}^{-\int_{s}^{t} g(u) \mathrm{d} u} c(s) G\left(x(s), x\left(s-r_{2}(s)\right)\right) \mathrm{d} s,
\end{aligned}
$$

where

$$
\mu(s)=\frac{\left(b^{\prime}(s)+b(s) g(s)\right)\left(1-r_{1}^{\prime}(s)\right)+r_{1}^{\prime \prime}(s) b(s)}{\left(1-r_{1}^{\prime}(s)\right)^{2}}
$$

Now we will present our main results. We set

$$
K=\sup _{t \geq 0}\left\{\mathrm{e}^{-\int_{0}^{t} g(u) \mathrm{d} u}\right\}
$$

Let $\psi \in C B([m(0), 0], E)$ be a fixed. Put

$$
p=\frac{U_{1}}{U_{2}},
$$

where

$$
\begin{gathered}
U_{1}=\left(1+\left|\frac{b(0)}{1-r_{1}^{\prime}(0)}\right|+\int_{-r_{2}(0)}^{0} g(u) \mathrm{d} u\right) K+L \int_{0}^{t} \mathrm{e}^{-\int_{s}^{t} g(u) \mathrm{d} u}|c(s)| \mathrm{d} s \\
U_{2}=1-\left[\left|\frac{b(t)}{1-r_{1}^{\prime}(t)}\right|+\int_{t-r_{1}(t)}^{t} g(u) \mathrm{d} u+\int_{0}^{t} \mathrm{e}^{-\int_{s}^{t} g(u) \mathrm{d} u} g(s)\left(\int_{s-r_{1}(s)}^{s} g(u) \mathrm{d} u\right) \mathrm{d} s\right. \\
\left.+\int_{0}^{t} \mathrm{e}^{-\int_{s}^{t} g(u) \mathrm{d} u}\left\{\left|g\left(s-r_{1}(s)\right)\left(1-r_{1}^{\prime}(s)\right)-a(s)-\mu(s)\right|\right\} \mathrm{d} s\right]
\end{gathered}
$$

and $U_{2}>0$, this mean that

$$
\begin{aligned}
& {\left[\left|\frac{b(t)}{1-r_{1}^{\prime}(t)}\right|+\int_{t-\eta_{1}(t)}^{t} g(u) \mathrm{d} u+\int_{0}^{t} \mathrm{e}^{-\int_{s}^{t} g(u) \mathrm{d} u} g(s)\left(\int_{s-\eta_{1}(s)}^{s} g(u) \mathrm{d} u\right) \mathrm{d} s\right.} \\
& \left.+\int_{0}^{t} \mathrm{e}^{-\int_{s}^{t} g(u) \mathrm{d} u}\left\{\left|g\left(s-r_{1}(s)\right)\left(1-r_{1}^{\prime}(s)\right)-a(s)-\mu(s)\right|\right\} \mathrm{d} s\right]<1 .
\end{aligned}
$$


Let us recall that for continuous function $f: R \rightarrow E$, the first order modulus of smoothness for $f$ is the function $\omega: R \rightarrow R_{+} \cup\{0\}$ defined for any $\sigma \geq 0$ by

$$
\omega(f, \sigma)=\sup \left\{\left\|f\left(t^{\prime}\right)-f\left(t^{\prime \prime}\right)\right\|: t^{\prime}, t^{\prime \prime} \in R,\left|t^{\prime}-t^{\prime \prime}\right| \leq \sigma\right\} .
$$

Define

$$
\begin{aligned}
S_{\psi}= & \{\varphi \in C B([m(0), \infty), E), \varphi(t)=\psi(t) \text { for } t \in[m(0), 0], \varphi(t) \rightarrow 0 \\
& \text { as } t \rightarrow \infty,\|\varphi(\cdot)\| \leq p,\|\varphi(t)-\varphi(z)\| \leq \omega(t-z)\} .
\end{aligned}
$$

Now, we use (6) to define the operator $P: S_{\psi} \rightarrow S_{\psi}$ by $(P \varphi)(t)=\psi(t)$ if $t \in[m(0), 0)$ and for $t \geq 0$ we let

$$
(P \varphi)(t)=\sum_{i=1}^{6} I_{i}(t)
$$

where

$$
\begin{gathered}
I_{1}=\left(\varphi(0)-\frac{b(0)}{1-r_{1}^{\prime}(0)} \varphi\left(-r_{1}(0)\right)-\int_{-\eta_{1}(0)}^{0} g(u) \varphi(u) \mathrm{d} u\right) \mathrm{e}^{-\int_{0}^{t} g(u) \mathrm{d} u}, \\
I_{2}=\frac{b(t)}{1-r_{1}^{\prime}(t)} \varphi\left(t-r_{1}(t)\right), \\
I_{3}=\int_{t-\eta_{1}(t)}^{t} g(u) \varphi(u) \mathrm{d} u, \\
I_{4}=\int_{0}^{t} \mathrm{e}^{-\int_{s}^{t} g(u) \mathrm{d} u} g(s)\left(\int_{s-r_{1}(s)}^{s} g(u) \varphi(u) \mathrm{d} u\right) \mathrm{d} s, \\
I_{5}=\int_{0}^{t} \mathrm{e}^{-\int_{s}^{t} g(u) \mathrm{d} u}\left[g\left(s-r_{1}(s)\right)\left(1-r_{1^{\prime}}(s)\right)-a(s)-\mu(s)\right] \varphi\left(s-r_{1}(s)\right) \mathrm{d} s, \\
I_{6}=\int_{0}^{t} \mathrm{e}^{-\int_{s}^{t} g(u) \mathrm{d} u} c(s) G\left(x(s), x\left(s-r_{2}(s)\right)\right) \mathrm{d} s, \text { for } t \geq 0,
\end{gathered}
$$

where

$$
\mu(s)=\frac{\left(b^{\prime}(s)+b(s) g(s)\right)\left(1-r_{1}^{\prime}(s)\right)+r_{1}^{\prime \prime}(s) b(s)}{\left(1-r_{1}^{\prime}(s)\right)^{2}} .
$$

Theorem 2 Assume that the function $G \in C B(E \times E, E)$ is uniformly continuous, satisfies the condition $G(0,0)=0$ and there exists a constant $0<k_{1}<1$ such that

$$
\alpha\left(G\left(V_{1}(J), V_{2}(J)\right)\right) \leq k_{1} \max \left\{\alpha\left(V_{1}(J)\right), \alpha\left(V_{2}(J)\right)\right\},
$$

for each $V_{1}, V_{2} \subset S_{\psi}$ and for each $J=[m(0), T], T \in[m(0), \infty)$, where $\alpha$ denotes the Kuratowski measure of noncompactness. Moreover, we assume that there exists a constant $p_{1} \in(0,1)$ satisfies, for $t \geq 0$ :

$$
\begin{aligned}
& k_{1}+\left[\frac{b(t)}{1-r_{1}^{\prime}(t)}+\int_{t-\eta_{1}(t)}^{t} g(u) \mathrm{d} u+\int_{0}^{t} \mathrm{e}^{-\int_{s}^{t} g(u) \mathrm{d} u} g(s)\left(\int_{s-\eta_{1}(s)}^{s} g(u) \mathrm{d} u\right) \mathrm{d} s\right. \\
& \left.+\int_{0}^{t} \mathrm{e}^{-\int_{s}^{t} g(u) \mathrm{d} u}\left\{\left|g\left(s-r_{1}(s)\right)\left(1-r_{1}^{\prime}(s)-a(s)-\mu(s)\right)\right|\right\} \mathrm{d} s\right] \leq p_{1},
\end{aligned}
$$


where

$$
\mu(s)=\frac{\left(b^{\prime}(s)+b(s) g(s)\right)\left(1-r_{1}^{\prime}(s)\right)+r_{1}^{\prime \prime}(s) b(s)}{\left(1-r_{1}^{\prime}(s)\right)^{2}}
$$

and $\int_{0}^{t} \mathrm{e}^{-\int_{s}^{t} g(u) \mathrm{d} u}|c(s)| \mathrm{d} s \rightarrow 0$, as $t \rightarrow \infty$.

Then there exists at least one solution of the problem (1), which tends to zero if

$$
\int_{0}^{t} g(s) \mathrm{d} s \rightarrow \infty, \text { as } t \rightarrow \infty
$$

Proof. We divide our proof into parts. In the first part, we show that $(P \varphi): S_{\psi} \rightarrow S_{\psi}$ complete with is well defined. Next, using Sadovskii's fixed point theorem and techniques of the theory of the measure of noncompactness, we prove, that there exists a fixed point of the operator $(P \varphi)$, which is a solution of the problem (1). In the last part of our proof, we will show, that the solution of (1) tends to zero as $t \rightarrow \infty$ if $\int_{0}^{t} g(s) \mathrm{d} s \rightarrow \infty$, as $t \rightarrow \infty$.

Part I. It is clear that $(P \varphi):[m(0), \infty) \rightarrow E$ is continuous. We will show the boundedness of this operator. Notice that $G \in C B(E \times E, E)$, so there exists a constant $L>0$ such that $\left\|G\left(x(t), x\left(t-r_{2}(t)\right)\right)\right\| \leq L, x \in S_{\psi}, t \in[m(0), \infty]$.

For $t \in[m(0), 0]$, the boundedness of the operator $(P \varphi)$ is clear. For $t>0$ we have

$$
\begin{aligned}
\|P \varphi(t)\| \leq \| & \left.\varphi(0)-\frac{b(0)}{1-r_{1}^{\prime}(0)} \varphi\left(-r_{1}(0)\right)-\int_{-r_{2}(0)}^{0} g(u) \varphi(u) \mathrm{d} u\right] \mathrm{e}^{-\int_{0}^{t} g(u) \mathrm{d} u} \\
& +\frac{b(t)}{1-r_{1}^{\prime}(t)} \varphi\left(t-r_{1}(t)\right)+\int_{t-r_{1}(t)}^{t} g(u) \varphi(u) \mathrm{d} u \\
& -\int_{0}^{t} \mathrm{e}^{-\int_{s}^{t} g(u) \mathrm{d} u} g(s)\left(\int_{s-r_{1}(s)}^{s} g(u) \varphi(u) \mathrm{d} u\right) \mathrm{d} s \\
& +\int_{0}^{t} \mathrm{e}^{-\int_{s}^{t} g(u) \mathrm{d} u}\left[g\left(s-r_{1}(s)\right)\left(1-r_{1}^{\prime}(s)-a(s)-\mu(s)\right)\right] \varphi\left(s-r_{1}(s)\right) \mathrm{d} s \\
& +\int_{0}^{t} \mathrm{e}^{-\int_{s}^{t} g(u) \mathrm{d} u} c(s) G\left(\varphi(s), \varphi\left(s-r_{2}(s)\right)\right) \mathrm{d} s \| \\
\leq & \left(1+\left|\frac{b(0)}{1-r_{1}^{\prime}(0)}\right|+\int_{-r_{2}(0)}^{0} g(u) \mathrm{d} u\right) \mathrm{e}^{-\int_{0}^{t} g(u) \mathrm{d} u} \\
& +\|\varphi\|\left|\frac{b(t)}{1-r_{1}^{\prime}(t)}\right|+\|\varphi\| \int_{t-r_{1}(t)}^{t} g(u) \mathrm{d} u \\
& +L \int_{0}^{t} \mathrm{e}^{-\int_{s}^{t} g(u) \mathrm{d} u}|c(s)| \mathrm{d} s \\
& +\|\varphi\| \int_{0}^{t} \mathrm{e}^{-\int_{s}^{t} g(u) \mathrm{d} u} g(s)\left(\int_{s-r_{1}(s)}^{s} g(u) \mathrm{d} u\right) \mathrm{d} s \\
& \| \int_{0}^{t} \mathrm{e}^{-\int_{s}^{t} g(u) \mathrm{d} u}\left\{g\left(s-r_{1}(s)\right)\left(1-r_{1}^{\prime}(s)-a(s)-\mu(s)\right)\right\} \mathrm{d} s
\end{aligned}
$$




$$
\begin{aligned}
\leq & \left(1+\left|\frac{b(0)}{1-r_{1}^{\prime}(0)}\right|+\int_{-r_{2}(0)}^{0} g(u) \mathrm{d} u\right) K \\
& +\|\varphi\|\left[\left|\frac{b(t)}{1-r_{1}^{\prime}(t)}\right|+\int_{t-\eta_{1}(t)}^{t} g(u) \mathrm{d} u+\int_{0}^{t} \mathrm{e}^{-\int_{s}^{t} g(u) \mathrm{d} u} g(s)\left(\int_{s-\eta_{1}(s)}^{s} g(u) \mathrm{d} u\right) \mathrm{d} s\right. \\
& \left.+\int_{0}^{t} \mathrm{e}^{-\int_{s}^{t} g(u) \mathrm{d} u}\left\{\left|g\left(s-r_{1}(s)\right)\left(1-r_{1}^{\prime}(s)-a(s)-\mu(s)\right)\right|\right\} \mathrm{d} s\right] \\
& +L \int_{0}^{t} \mathrm{e}^{-\int_{s}^{t} g(u) \mathrm{d} u}|c(s)| \mathrm{d} s,
\end{aligned}
$$

where

$$
\mu(s)=\frac{\left(b^{\prime}(s)+b(s) g(s)\right)\left(1-r_{1}^{\prime}(s)\right)+r_{1}^{\prime \prime}(s) b(s)}{\left(1-r_{1}^{\prime}(s)\right)^{2}} .
$$

Therefore, because $\varphi \in S_{\psi}$ satisfies $\|\varphi(\cdot)\| \leq p$, we have, by (9), that $\|P \varphi(\cdot)\| \leq p$.

Now we will show that for each $\varphi \in S_{\psi}$ we obtain

$$
\forall \epsilon>0 \exists \delta>0|t-z|<\delta \Rightarrow\|(P \varphi)(t)-(P \varphi)(z)\|<\epsilon .
$$

Let's note that

$$
\|(P \varphi)(t)-(P \varphi)(z)\| \leq \sum_{i=1}^{6}\left\|A_{i}(t)-A_{i}(z)\right\|
$$

where

$$
\begin{aligned}
& A_{1}=\left(\varphi(0)-\frac{b(0)}{1-r_{1}^{\prime}(0)} \varphi\left(-r_{1}(0)\right)-\int_{-r_{1}(0)}^{0} g(u) \varphi(u) \mathrm{d} u\right)\left(\mathrm{e}^{-\int_{0}^{t} g(u) \mathrm{d} u}-\mathrm{e}^{-\int_{0}^{z} g(u) \mathrm{d} u}\right), \\
& A_{2}=\frac{b(t)}{1-r_{1}^{\prime}(t)} \varphi\left(t-r_{1}(t)\right)-\frac{b(z)}{1-r_{1}^{\prime}(z)} \varphi\left(z-r_{1}(z)\right), \\
& A_{3}=\int_{t-\eta_{1}(t)}^{t} g(u) \varphi(u) \mathrm{d} u-\int_{z-\eta_{1}(z)}^{z} g(u) \varphi(u) \mathrm{d} u, \\
& A_{4}=\int_{0}^{t}\left(\mathrm{e}^{-\int_{s}^{t} g(u) \mathrm{d} u}-\mathrm{e}^{-\int_{s}^{z} g(u) \mathrm{d} u}\right) g(s)\left(\int_{s-r_{1}(s)}^{s} g(u) \varphi(u) \mathrm{d} u\right) \mathrm{d} s, \\
& A_{5}=\int_{0}^{t}\left(\mathrm{e}^{-\int_{s}^{t} g(u) \mathrm{d} u}-\mathrm{e}^{-\int_{s}^{z} g(u) \mathrm{d} u}\right)\left[g\left(s-r_{1}(s)\right)\left(1-r_{1}^{\prime}(s)\right)-a(s)-\mu(s)\right] \varphi\left(s-r_{1}(s)\right) \mathrm{d} s, \\
& A_{6}=\int_{0}^{t}\left(\mathrm{e}^{-\int_{s}^{t} g(u) \mathrm{d} u}-\mathrm{e}^{-\int_{s}^{z} g(u) \mathrm{d} u}\right) c(s) G\left(\varphi(s), \varphi\left(s-r_{2}(s)\right)\right) \mathrm{d} s .
\end{aligned}
$$

Because the exponential function is continuous and using assumptions about functions $\varphi, g, b$ and properties of Bochner integral we have

$$
\forall \epsilon>0 \exists \delta_{1}>0|t-z|<\delta_{1} \Rightarrow\left\|A_{1}(t)-A_{1}(z)\right\|<\frac{1}{6} \epsilon .
$$

By equicontinuity of function $G$ and equicontinuity of family $S_{\psi}$ we have that

$$
\forall \epsilon>0 \exists \delta_{2}>0|t-z|<\delta_{2} \Rightarrow\|G(\varphi(t), \varphi(t-\tau(t)))-G(\varphi(z), \varphi(z-\tau(z)))\|<\frac{1}{6} \epsilon .
$$


So we obtain that $\left\|A_{2}(t)-A_{2}(z)\right\|<\frac{1}{6} \epsilon$.

In the same way, we estimate the remaining ingredients and we get

$$
\forall \epsilon>0 \exists \delta>0|t-z|<\delta \Rightarrow\|(P \varphi)(t)-(P \varphi)(z)\|<\epsilon .
$$

Now we will show, that $(P \varphi)(t) \rightarrow 0$ as $t \rightarrow \infty$. It is obvious that the first term $I_{1}(t)$ tends to zero as $t \rightarrow \infty$, by the condition (13). Because $\varphi\left(t-r_{1}\right) \rightarrow 0$ and $t-r_{1}(t) \rightarrow \infty$ as $t \rightarrow \infty$ and $b$ is a bounded function so the second term $I_{2}(t)$ tends to zero as $t \rightarrow \infty$. Because functions $\varphi, g$ are bounded functions, so $I_{3} \rightarrow 0, t \rightarrow \infty$. Analogously $I_{4}$ and $I_{5}$ tends to zero if $t \rightarrow \infty$. Moreover,

$$
\begin{aligned}
\left\|A_{6}\right\| & \leq\left\|\int_{0}^{t} \mathrm{e}^{-\int_{s}^{t} g(u) \mathrm{d} u} c(s) G\left(x(s), x\left(s-r_{2}(s)\right)\right) \mathrm{d} s\right\| \\
& \leq L \int_{0}^{t} \mathrm{e}^{-\int_{s}^{t} g(u) \mathrm{d} u}|c(s)| \mathrm{d} s \rightarrow 0, \quad t \rightarrow \infty .
\end{aligned}
$$

In conclusion $(P \varphi)(t) \rightarrow 0$ as $t \rightarrow \infty$. Hence $P_{\varphi}$ maps $S_{\psi}$ into $S_{\psi}$.

Part II. Suppose that $V \subset S_{\psi}$ and $\alpha$ denotes the Kuratowski measure of noncompactness. Let $J=[m(0), T], T \in[m(0), \infty)$. Then,

$$
\begin{aligned}
& \alpha(P(V)(t)) \\
& \leq \alpha(V(J)) \cdot\left[\frac{b(t)}{1-r_{1}^{\prime}(t)}+\int_{t-\eta_{1}(t)}^{t} g(u) \mathrm{d} u\right. \\
& +\int_{0}^{t} \mathrm{e}^{-\int_{s}^{t} g(u) \mathrm{d} u} g(s)\left(\int_{s-\eta_{1}(s)}^{s} g(u) \mathrm{d} u\right) \mathrm{d} s \\
& \left.+\int_{0}^{t} \mathrm{e}^{-\int_{s}^{t} g(u) \mathrm{d} u}\left\{g\left(s-r_{1}(s)\right)\left(1-r_{1}^{\prime}(s)\right)-a(s)-\mu(s)\right\} \mathrm{d} s\right] \\
& +k_{1} \cdot \max \left\{\alpha\left(V_{1}\right), \alpha\left(V_{2}\right)\right\} \\
& \leq \alpha(V(J)) \cdot\left[\frac{b(t)}{1-r_{1}^{\prime}(t)}+\int_{t-\eta_{1}(t)}^{t} g(u) \mathrm{d} u\right. \\
& +\int_{0}^{t} \mathrm{e}^{-\int_{s}^{t} g(u) \mathrm{d} u} g(s)\left(\int_{s-\eta_{1}(s)}^{s} g(u) \mathrm{d} u\right) \mathrm{d} s \\
& \left.+\int_{0}^{t} \mathrm{e}^{-\int_{s}^{t} g(u) \mathrm{d} u}\left\{\left|g\left(s-r_{1}(s)\right)\left(1-r_{1}^{\prime}(s)\right)-a(s)-\mu(s)\right|\right\} \mathrm{d} s+k_{1}\right] \\
& \leq p_{1} \cdot \alpha(V(J)) .
\end{aligned}
$$

So, using (12) and properties of $\alpha$ we have

$$
\alpha(V(J))=\sup _{t \in J} \alpha(V(t))=\sup _{t \in J} \alpha(P(V)(t)) \leq p_{1} \cdot \alpha(V(J)) .
$$

Because $p_{1}<1$, we get $\alpha(V(t))=0$ for each $t \in J$.

Define $\Phi(V)=\left(\sup _{t \in J_{1}} \alpha(V(t)), \sup _{t \in J_{2}} \alpha(V(t)), \cdots\right)$ for any nonempty subset $V$ of $S_{\psi}$, where $J_{k}=[m(0), k], k=1,2, \cdots, n$. Evidently $\Phi(V) \in S_{\infty}$. By the properties of $\alpha$, the function $\Phi$ satisfies conditions (2)-(4). From (10) $\Phi(P(V))<\Phi(V)$, whenever $\Phi(V)>0$. If $\Phi(V)=0$ then for each $t \in[m(0), \infty)$ we have $\alpha(V(t))=0$. Hence Arzela-Ascoli's theorem proves that the set $V$ is relatively compact. Consequently, by Theorem 1 , the operator 
$(P \varphi)$ has a fixed point which is a solution of the problem (1) with $x(t)=\Phi(t)$ on $[m(0), 0]$ and $x(t) \rightarrow 0$ as $t \rightarrow \infty$, if $\int_{0}^{t} g(s) \mathrm{d} s \rightarrow \infty$, for $t \rightarrow \infty$. The proof is complete.

Remark 1. It is clear that we can assume about the function $G$ other types of continuity and other conditions on measures of noncompactness. When we investigate the existence of solutions of (1) with non-continuous right-hand side, it is natural to consider the so-called Carathéodory-type solutions.

\section{Conflicts of Interest}

The authors declare no conflicts of interest regarding the publication of this paper.

\section{References}

[1] Burton, T.A. (2006) Stability for Fixed Point Theory for Functional Differential Equations. Dover Publications, New York.

[2] Becker, L.C. and Burton, T.A. (2006) Stability, Fixed Points and Inverse of Delay, Proceedings of the Royal Society of Edinburgh Section A: Mathematics, 136, 245-275. https://doi.org/10.1017/S0308210500004546

[3] Ardjouni, A. and Djoudi, A. (2012) Fixed Points and Stability in Neutral Nonlinear Differential Equations with Variable Delays. Opuscula Mathematica, 32, 5-19.

https://doi.org/10.7494/OpMath.2012.32.1.5

[4] Jin, C.-H. and Luo, J.-W. (2008) Stability in Functional Differential Equations Established Using Fixed Point Theory. Nonlinear Analysis: Theory, Methods \& Applications, 68, 3307-3315. https://doi.org/10.1016/j.na.2007.03.017

[5] Burton, T.A. (2001) Liapunov Functional, Fixed Points, and Stability by Krasnoselskii's Theorem. Nonlinear Studies, 9, 181-190.

[6] Burton, T.A. (2003) Stability by Fixed Point Theory or Liapunov's Theory: A comparison. International Journal on Fixed Point Theory Computation and Applications, 4, 15-32.

[7] Ardjouni, A. and Djoudi, A. (2011) Fixed Points and Stability in Linear Neutral Differential Equations with Variable Delays. Nonlinear Analysis. Theory, Methods \& Applications, 74, 2062-2070. https://doi.org/10.1016/j.na.2010.10.050

[8] Ardjouni, A. and Djoudi, A. (2011) Stability in Nonlinear Neutral Differential Equations with Variable Delays Using Fixed Point Theory. Electronic Journal of Qualitative Theory of Differential Equations, No. 43, 1-11.

https://doi.org/10.14232/ejqtde.2011.1.43

[9] Burton, T.A. (2004) Fixed Points and Stability of Nonconvolution Equation. Proceedings of the American Mathematical Society, 132, 3679-3687. https://doi.org/10.1090/S0002-9939-04-07497-0

[10] Burton, T.A. and Furumochi, T. (2001) A Note on Stability by Schauder's Theorem, Funkcialaj Ekvacioj, 44, 73-82.

[11] Burton, T.A. and Furumochi, T. (2001) Fixed Points and Problems in Stability Theory. Dynamical Systems and Applications, 10, 89-116.

[12] Burton, T.A. and Furumochi, T. (2002) Asymptotic Behavior of Solutions of Functional Differential Equations by Fixed Point Theorems. Dynamic Systems and Applications, 11, 499-519. 
[13] Burton, T.A. and Furumochi, T. (2002) Krasnosielskii's Fixed Point Theorem and Stability. Nonlinear Analysis: Theory, Methods \& Applications, 49, 445-454. https://doi.org/10.1016/S0362-546X(01)00111-0

[14] Hale, J.K. (1977) Theory of Functional Differential Equations. Springer-Verlag, New York. https://doi.org/10.1007/978-1-4612-9892-2

[15] Jin, C.-H. and Luo, J.-W. (2008) Fixed Points and Stability in Neutral Differential Equations with Variable Delays. Proceedings of the American Mathematical Society, 136, 909-918. https://doi.org/10.1090/S0002-9939-07-09089-2

[16] Raffoul, Y.N. (2004) Stability in Neutral Nonlinear Differential Equations with Functional Delays Using Fixed-Point Theory. Mathematical and Computer Modelling, 40, 691-700. https://doi.org/10.1016/j.mcm.2004.10.001

[17] Zhang, B. (2005) Fixed Points and Stability in Differential Equations with Variable Delays. Nonlinear Analysis: Theory, Methods \& Applications, 63, 233-242. https://doi.org/10.1016/j.na.2005.02.081

[18] Sadovskii, B.N. (1972) Limit-Compact and Condensing Operators. Russian Mathematical Surveys, 27, 86-144. https://doi.org/10.1070/RM1972v027n01ABEH001364

[19] Boe, E. and Chang, H.-C. (1989) Dynamics of Delayed Systems under Feedback Control. Chemical Engineering Science, 44, 1281-1294. https://doi.org/10.1016/0009-2509(89)85002-X

[20] Brayton, R.K. and Willoughby, R.A. (1976) On the Numerical Integration of a Symmetric System of Difference-Differential Equations of Neutral Type. Journal of Mathematical Analysis and Applications, 18, 182-189. https://doi.org/10.1016/0022-247X(67)90191-6

[21] Driver, D.R. (1984) A Mixed Neutral Systems. Nonlinear Analysis. Theory, Methods \& Applications, 8, 155-158. https://doi.org/10.1016/0362-546X(84)90066-X

[22] Popov, E.P. (1966) Automatic Regulation and Control. Nauka, Moscow, Russian.

[23] Banaś, J. and Goebel, K. (1980) Measures of Noncompactness in Banach Spaces. Mercel Dekker, New York.

[24] Ambrosetti, A. (1967) Un teorema di esistenza per le equazioni diffenziali negli spazi di Banach. Rendiconti del Seminario Matematico della Università di Padova, 39, 349-360. 